\title{
Profile modification by light pressure in plasmas expanding with uniform, time-dependent temperature
}

\author{
J. R. Sanmartín and J. L. Montañes \\ Escuela Técnica Superior de Ingenieros Aeronáuticos, Universidad Politécnica de Madrid, Madrid, Spain \\ (Received 16 October 1979; accepted 20 August 1980)
}

Profile modification of laser plasmas, in the transition layer at critical density and in the flow on the overdense side, is studied. Assuming isothermal flow and low absorption within the layer, compression transitions are proved impossible and cavities possible only in subsonic flow. The overdense flow adjusts itself for a rarefaction transition in a manner (formation of plateaus, bumps, or cavities) critically dependent on how the (spatially uniform) temperature varies with time. Spherical effects and evidence for the results are considered.

\section{INTRODUCTION}

Radiation pressure-induced profile steepening at the critical density $n_{c}$, has been observed in laser-produced plasmas and may affect the absorption processes. ${ }^{1}$ There have been theoretical analyses of the flow through the steep critical layer and the transition of the laser wave from evanescent (overdense side) to oscillatory (underdense side). 2-5 Density plateaus, ${ }^{4,6,7}$ cavities, ${ }^{8,9}$ and bumps, ${ }^{5,7}$ in addition to steepening, have been observed in simulations and experiments, sometimes in the same pulse. $1,6,10$

In this paper we prove that, assuming isothermal flow and neglecting absorption in the transition layer, 2,4 compression front structures are impossible and cavities may only appear in subsonic flow. We then study how the radiation pressure affects the overdense region of the expansion flow, where the laser light does not reach. Such an indirect effect must exist. The structure of a rarefaction front transition leads to a relation between density and Mach number (in a frame moving with the layer) at its overdense side. ${ }^{4}$ (Compression and rarefaction fronts are sometimes called $R$ and $D$ fronts..$^{3,11}$ ) Since the flow, in the absence of radiation steepening, will not satisfy that relation at an arbitrary density (that is, arbitrary incident field), the overdense plasma needs to adjust. We find that the adjustment occurs and depends nontrivially on how the electron temperature $T_{e}$ changes in time, explaining, we believe, the differing observations mentioned previously ${ }^{1,4-10}$ and why sometimes plateaus did not form when expected. ${ }^{5,9}$

In our analysis we assume normal incidence (or $s$ polarization), cold ions, and quasi-neutral flow ${ }^{4}$ and neglect the $T_{e}$ gradient ${ }^{2,4}$ (retaining the time dependence) in the momentum equation throughout the overdense flow; this applies strictly to such high laser intensities that a thermal wave heats the high-density target, ${ }^{12}$ and fails at lower intensities near the ablation surface. A uniform $T_{e}$ follows at high intensities from large thermal and radiation conduction terms in the energy equation. We ignore this equation entirely, leaving $T_{e}(t)$, which could follow from an overall energy balance, undetermined. For simplicity, we consider only time power laws, $T_{e} \sim t^{\alpha}$ and planar geometry (initially, the target of the laser pulse fills the halfspace $x>0$ ).
We expect the general features of our results to apply to conditions broader than those considered, because their basis is the behavior of rarefaction waves with spatially uniform temperature, in the absence of radiation. We find that the well-known case where the temperature is also constant in time, ${ }^{13}$ extensively used in laser-plasmas analyses, ${ }^{14}$ is a very singular limit; when a temperature time dependence is allowed for, nontrivial changes occur in the flow which affect the adjustment to profile steepening. We finally note that planar results for a given $\alpha$ may be roughly used in a spherical problem with some larger $\alpha$.

\section{BASIC EQUATIONS}

For a quasi-neutral plasma expanding in planar geometry, with negligible ion temperature and no radiation pressure, the density and momentum conservation equations for the ion-electron fluid are

$$
\begin{aligned}
& \frac{\partial n}{\partial t}+\frac{\partial n v}{\partial x}=0, \\
& \frac{\partial n v}{\partial t}+\frac{\partial n v^{2}}{\partial x}+\frac{\partial n c_{T}^{2}}{\partial x}=0,
\end{aligned}
$$

where $c_{T}^{2}=T_{e} / m$ and $v$ and $m$ are ion velocity and mass. Radiation pressure adds to Eq. (2) an appropriate component of $\nabla \cdot P_{r}$ where ${ }^{15}$

$$
\mathrm{P}_{r}=1 \frac{\left\langle E^{2}+B^{2}\right\rangle}{8 \pi}-\frac{\left\langle\epsilon_{\mathrm{Re}} \mathrm{EE}+\mathrm{BB}\right\rangle}{4 \pi} ;
$$

$\mathrm{E}$ and $\mathrm{B}$ are electric and magnetic fields, $\epsilon_{\mathrm{R}_{\theta}}$ is the real part of the dielectric function and the average is over a wave period $2 \pi / \omega$, typically less than the characteristic flow time $\tau$ by a factor of $10^{-6}$. For normal incidence (extension to $s$ polarization is trivial), and setting $E=\operatorname{Re}\left(\hat{E}_{e}^{i \omega t}\right), \hat{E}$ complex, Eq. (2) becomes

$$
\frac{\partial n v}{\partial t}+\frac{\partial}{\partial x}\left(n v^{2}+n c_{T}^{2}+\frac{|\hat{E}|^{2}+|c \partial \hat{E} / \omega \partial x|^{2}}{16 \pi m}\right)=0 .
$$

In addition we have the wave equation

$$
\frac{\partial^{2} \hat{E}}{\partial x^{2}}+\left(\frac{\omega^{2}}{c^{2}}\right) \epsilon \hat{E}=0 .
$$

Using (4), the radiation pressure term in $\left(2^{\prime}\right)$ trans forms to (* stands for the complex conjugate) 


$$
\frac{1-\epsilon_{R_{\theta}}}{16 \pi} \frac{\partial|\hat{E}|^{2}}{\partial x}-\frac{i \epsilon_{I m}}{16 \pi}\left(\hat{E} \frac{\partial \hat{E}^{*}}{\partial x}-\hat{E}^{*} \frac{\partial \hat{E}}{\partial x}\right) .
$$

Assuming $\epsilon_{\mathrm{Im}} \simeq 0$ (no absorption), taking $\epsilon=\epsilon_{\mathrm{R}}$ $\simeq 1-n / n_{c}$, and using (5) in $\left(2^{\prime}\right)$, we obtain a third conservation equation

$$
\frac{\partial v}{\partial t}+\frac{\theta}{\partial x}\left(\frac{v^{2}}{2}+c_{T}^{2} \ln n+\frac{|\hat{E}|^{2}}{16 \pi m n_{c}}\right)=0,
$$

which may be used instead of (4). Clearly, $\hat{E}$ may now be taken real. Notice that unless $\epsilon_{\mathrm{Im}_{\mathrm{m}}}=0$, the radiation force per unit volume is not $\left(-n / n_{c}\right) \nabla\left(|\hat{E}|^{2} / 16 \pi\right)$.

\section{TRANSITION AT THE CRITICAL LAYER}

We shall first examine the critical layer and prove that transitions other than that of Ref. 4 are impossible, although we find that cavities may appear in subsonic flow. Equations (1), $\left(2^{\prime}\right)$, and (6) involve two length scale $s / \omega$ and $c_{T} \tau$. Usually, $c_{T} \tau \omega / c \gg 1$; then, the transition occurs at a certain $x_{4}(t)$ in the long scale, and in a frame moving with $x_{c}$ conditions are quasisteady. ${ }^{3,4}$ Defining

$$
\begin{aligned}
& \xi=\left(x-x_{c}\right) \omega / c, \nu=n / n_{c}, \\
& \tilde{M}=\left(\dot{x}_{c}-v\right) / c_{T}, \mathcal{E}=\hat{E} /\left(4 \pi m n_{c} c_{T}^{2}\right)^{1 / 2},
\end{aligned}
$$

Eqs. (1), (2'), and (6) yield

$$
\begin{aligned}
& \nu \tilde{M}=\text { const }=\nu_{2} \tilde{M}_{2}=\nu_{1} \tilde{M}_{1}=\nu_{s} \bar{M}_{s}, \\
& \nu\left(1+\tilde{M}^{2}\right)+\frac{1}{4} \mathcal{E}^{2}+\frac{1}{4}(\partial \mathcal{E} / \partial \xi)^{2}=\mathrm{const}, \\
& =\nu_{2}\left(1+\tilde{M}_{2}^{2}\right)=\nu_{s}\left(1+\tilde{M}_{s}^{2}\right)+\frac{1}{4} \mathcal{E}_{s}^{2}, \\
& g(\tilde{M})+\frac{1}{2} \mathcal{E}^{2}=\text { const }=g\left(\tilde{M}_{2}\right)=g\left(\tilde{M}_{1}\right)=g\left(\tilde{M}_{s}\right)+\frac{1}{2} \mathcal{E}_{s}^{2},
\end{aligned}
$$

where $g(\tilde{M}) \equiv \tilde{M}^{2}-\ln \tilde{M}^{2}-1$ decreases from infinity at $\tilde{M}=0$ to zero at $\tilde{M}=1$, and then grows to infinity at $\bar{M}=\infty$. Equation (7) has been used to write (8) and (9) conveniently. The subscripts 1 and $s$ refer to nodes and maxima in the standing wave on one side of the layer, and 2 refers to conditions far on the evanescent wave at the other side; $\delta_{2}=\mathcal{E}_{1}=\partial \mathscr{E} /\left.\partial \xi\right|_{2}=\partial \mathscr{E} /\left.\partial \xi\right|_{s}=0$.

(a) First, consider the transitions $\tilde{M}_{2}<1, \bar{M}_{1}>1$, and $\bar{M}_{2}>1, \tilde{M}_{1}<1$. In either case, $\bar{M}=1$ at some point; since $g(\tilde{M})$ has minima at the maxima of $\mathcal{E}$, we have $\tilde{M}_{s}=1$, then,

$$
\left(\frac{\partial \mathcal{E}}{\partial \xi}\right)^{2}=4 \frac{(1-\tilde{M})^{2}}{\tilde{M}}\left(\frac{\tilde{M} g(\tilde{M})}{2(1-\tilde{M})^{2}}-\frac{\tilde{M}_{2} g\left(\tilde{M}_{2}\right)}{2\left(1-\tilde{M}_{2}\right)^{2}}\right) .
$$

Now, $\tilde{M} g(\tilde{M}) / 2(1-\tilde{M})^{2}$ is a monotonically increasing function of $\tilde{M}$ for $0<\tilde{M}<\infty$. Thus, conditions $\tilde{M}_{2}<1$, $\bar{M}_{1}>1$, those studied in Ref. 4 , are indeed possible [right-hand side of (10) positive], while conditions $\tilde{M}_{2}>1$, $\tilde{M}_{1}<1$ are not [right-hand side negative].

(b) The case $\tilde{M}_{2}>1, \tilde{M}_{1}>1$ is also impossible. Here $\tilde{M}_{1}=\tilde{M}_{2}$ and $1<\tilde{M}_{s}<\tilde{M}_{2} ;$ hence $\nu_{s}=\nu_{2} \tilde{M}_{2} / \tilde{M}_{s}>\nu_{2}$. On the other hand, $\mathcal{E}$ and $\partial^{2} \mathcal{E} / \partial \xi^{2}$ must have the opposite sign near $\mathcal{E}_{s}$, and the same sign in the evanescent tail, that is, from the wave equation, we get $\nu_{2}>1, \nu_{s}<1$, in contradiction to the preceding inequality. The argument does not apply to the case $\tilde{M}_{2}<1, \tilde{M}_{1}<1$. However, we again have $\tilde{M}_{1}=\tilde{M}_{2}$ : conditions at 1 are just those at 2 , and the wave is aIso evanescent on side $1\left(\partial \mathcal{E} /\left.\partial \xi\right|_{1}=0\right)$; the transition is not one in the sense we considered, and describes a cavity with a nonperiodic field isolated from the incident wave.

We conclude that there may be no $R$ fronts and that the $D$-front structure is entirely described by Eqs. (7) (9) together with $\tilde{M}_{s}=1$; in particular, ${ }^{4}$

$$
g\left(\tilde{M}_{2}\right)=\frac{\mathcal{E}_{s}^{2}}{2}, \nu_{2}=\frac{g\left(\tilde{M}_{2}\right)}{2\left(1-\tilde{M}_{2}\right)^{2}}, \tilde{M}_{2}<1 .
$$

The relation between $\mathcal{E}_{s}$ and the incident field can only be obtained by an analysis of the underdense region; an WKB approximation was used in Ref. 4 (see comment at the end).

\section{OVERDENSE FLOW MODIFICATION FOR SPATIALLY UNIFORM TEMPERATURE}

Now consider the overdense region. Here, there is no laser radiation, and we just have Eqs. (1) and (2). If $T_{e} \sim t^{\alpha}$, these equations have a self-similar solution, and the analysis is simplified. Introducing

$$
\eta=x / \int_{0}^{t} c_{x}\left(t^{\prime}\right) d t^{\prime}, \nu(\eta)=n / n_{c}, \quad u(\eta)=\nu / c_{T},
$$

we get

$$
\begin{aligned}
& \frac{d \ln \nu}{d \eta}=\frac{1}{\eta-u} \frac{d u}{d \eta}, \\
& {\left[(\eta-u)^{2}-1\right] \frac{d u}{d \eta}=\frac{\alpha u}{2+\alpha}(\eta-u) .}
\end{aligned}
$$

\section{A. Expansion flow in the absence of radiation}

Let us begin studying the expansion when laser radiation is neglected everywhere. Then, Eqs. (12) and (13). describe a rarefaction wave, the head of which is a weak discontinuity that advances into the undisturbed plasma with the sound velocity $\left(c_{r}\right.$, for $T_{e}$ uniform); the $\alpha=0$ case is the known isothermal expansion ${ }^{13}$ used extensively in laser-plasma interaction analyses. Hence, at $\eta=1$ we have $u=0, \nu=n_{0} / n_{c}\left(n_{0}\right.$, density in the undisturbed plasma). In addition, Eq. (12) requires $\nu(\eta-u)$, which vanishes with $\nu$, to increase monotonically with $\eta$; thus, $\eta-u>0$ (except possibly when $\nu=0$ ).

For $\alpha=0$, the integral curves of (13) are $u=$ const (giving $\nu=$ const) and $\eta-u= \pm 1[\mathrm{~F} \mathrm{ig.} \mathrm{1(a)].} \mathrm{Clearly,} \mathrm{the}$ solution is ${ }^{13}$

$$
\eta-u=1, \quad \nu=\left(n_{0} / n_{c}\right) \exp (\eta-1) .
$$

Figure 1(b) shows the integral curves for $\alpha<0$; then, the point $u=0, \eta=1$ is a saddle point, and the solution is one of the two integral curves through it (the other one is $u=0$ ). For $\alpha>0$, the point $u=0, \eta=1$ is a node; the solution belongs to the family of integral curves crossing the node with a common slope (the exceptional slope corresponds to $u=0$ ), and separates the integral curves such as I, that would lead to a multivalued solution, from those such as II, that reach the line $\eta-u=0$ with finite $\nu$ [Fig. 1(c)].

Notice that the plasma flows through a constant density (constant $\eta$ ) plane, at a (relative) Mach number given by $\eta-u$; for instance, the flow is sonic for $\alpha=0$. Now, assuming temperature uniform implies that heat 

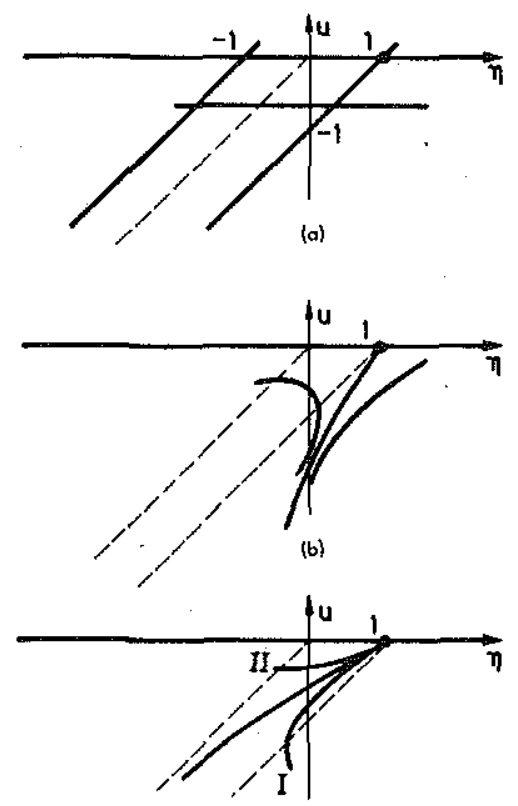

(c)

FIG. 1. Integral curves of Eq. (13) for a rarefaction wave with temperature $T_{c} \sim t^{\alpha}$; (a) $\alpha=0$; (b) $\alpha<0$; (c) $\alpha>0$. Dimensionless velocity $u$ and position $\eta$ defined in the text.

conduction is able to adjust the temperature instantaneously everywhere; then, clearly, for $\alpha<0$ the inertial lag in the velocity field, which is related to previous, larger temperatures, explains the supersonic $(\eta-u>1)$ character of the solution [Fig. 1(b)]. A similar explanation applies to the subsonic $(\eta-u<1)$ flow for $\alpha>0$.

\section{B. Radiation pressure effect}

We can now understand how in the presence of laser radiation, the overdense flow adjusts itself to meet conditions (11) [that is, $\left.\tilde{M}_{2}\left(\delta_{s}\right), \nu_{2}\left(\delta_{s}\right)\right]$ differently depending on whether $d T_{e} / d t \gtrless 0$. If the transition occurs at $\eta_{c}$, we have $\dot{x}_{c}=\eta_{c} c_{T}$; notice that at $\eta_{c}$, the relative Mach number $M \equiv \eta-u$ is just $\vec{M}$. For $\alpha=0$, the solution follows the integral curve $\eta-u=1\left[\mathrm{Fig}, 1\right.$ (a)] up to $\eta^{*}$, where it goes over to the integral curve $u=u^{*}\left(=\eta^{*}-1\right)$ until $\eta_{c}$ when the transition occurs [see Fig. 2(a)]. The values of $\eta^{*}$ and $\eta_{c}$ are then given by $\left(n_{0} / n_{c}\right) \exp \left(\eta^{*}-1\right)$ $=\nu_{2}, \eta_{c}-\left(\eta^{*}-1\right)=\bar{M}_{2} ; u=$ const yields $\nu=$ const, and, therefore, a plateau appears from $\eta^{*}$ to $\eta_{c}$.
For $\alpha<0\left(d T_{e} / d t<0\right)$, the solution is the one without radiation [call it $\left.\nu_{0}(\eta), M_{0}(\eta)\right]$, until $\eta^{*}$, when a shock brings it to point " $b$ " such that $M_{b}=1 / M_{a}$ (isothermal shock $\left.{ }^{13}\right), \nu_{b}=\nu_{a} M_{a}^{2}$, where $M_{a}=M_{0}\left(\eta^{*}\right), \nu_{a}=\nu_{0}\left(\eta^{*}\right)$. The solution, then, goes along the integral curve through point " $b$ " until $\eta=\eta_{c}$, when the transition occurs [Fig. 2(b)]. Both $\eta^{*}$ and $\eta_{\mathfrak{c}}$ follow from the requirement that $\nu=\nu_{2}$ and $M=\tilde{M}_{2}$ at $\eta_{c}$. We find that this solution does not exist unless $\mathcal{E}_{s}$ exceeds a threshold, which corresponds to the limiting case $\eta^{*}=\eta_{c}$, and is shown in Fig. 3. A solution without a shock is impossible (it would imply a transition with $\left.\tilde{M}_{2}>1\right)$. For $\alpha>0\left(d T_{e} / d t>0\right)$, the solution up to $\eta_{c}$ is an integral curve (through the node), different from the one for negligible radiation, such that $\nu=\nu_{2}$ for $M=\tilde{M}_{2}$ [F ig. 2(c)].

\section{DISCUSSION}

We have found that a plateau (a region of density constant in the long scale) develops if, and only if, $d T_{e} / d t$ $\simeq 0$. Plateaus were indeed observed in simulations satisfying this condition. 4,6 A simple prediction of the theory is the length of the plateau: the ratio between this length and that of the density exponential decay, in Fig. 2(a), is $\left(1-M_{2}\right) / \ln \left(n_{0} \nu_{2} / n_{c}\right) ;$ for $n_{0} / n_{c}=4$ and $\nu_{2}$ $\simeq 1.5\left(M_{2} \simeq 0.32\right)$, the case of Figs. $1(\mathrm{a})$ and $1(\mathrm{~d})$ of Ref. 4 , the ratio is about 1.4, while the figures yield 1.6, a fair agreement.

A shock develops for $d T_{e} / d t<0$, if the electric field exceeds a threshold (Fig. 3); we found no smooth solution to the wave-plasma equations below the threshold. The compressed region between the shock and an usual $D$ front forms a bump. ${ }^{3}$ Condition $d T_{e} / d t<0$ is unusual during the pulse, and no shock observations are known to the authors. However, we have verified, that in spherical geometry, supersonic (relative to constant density surfaces) flow, leading to shocks, exists for $T_{e}$ constant or increasing moderately, as for instance, in Figs. 2 and 3 of Ref. 5 (see also Ref. 7): Notice that (i) the bump is several wavelengths wide so that radiation should be negligible in the back; (ii) the plasma enters the back supersonically and leaves it subsonically; (iii) the product of the Mach numbers is about 0.95 in fair agreement with the unity value corresponding to an isothermal shock.

Neither plateaus nor (overdense) shocks develop for $d T_{e} / d t>0$. For this condition, results from experi-
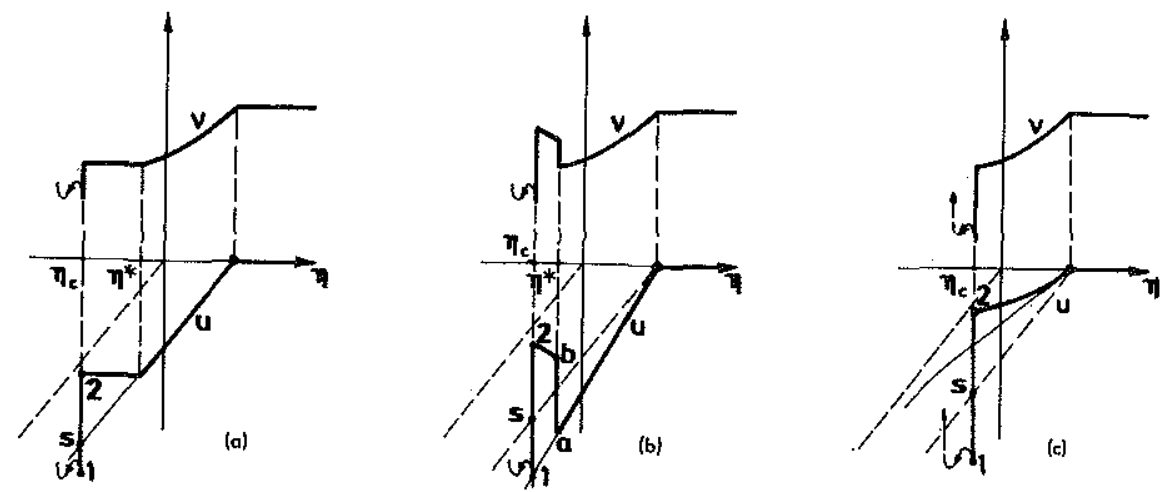

FIG. 2. Overdense flow for $T_{e} \sim t^{\alpha}$, modified by transition $2 \rightarrow 1$ ( $s-$ sonic point); (a) $\alpha=0$; (b) $\alpha^{\prime}<0$ (shock " $a$ " " $b$ " at $\left.\eta^{*}\right) ;$ (c) $\alpha>0$. Dimensionless density $\nu$ defined in the text; wavy lines: underdense flow. Light is incident from the left. 


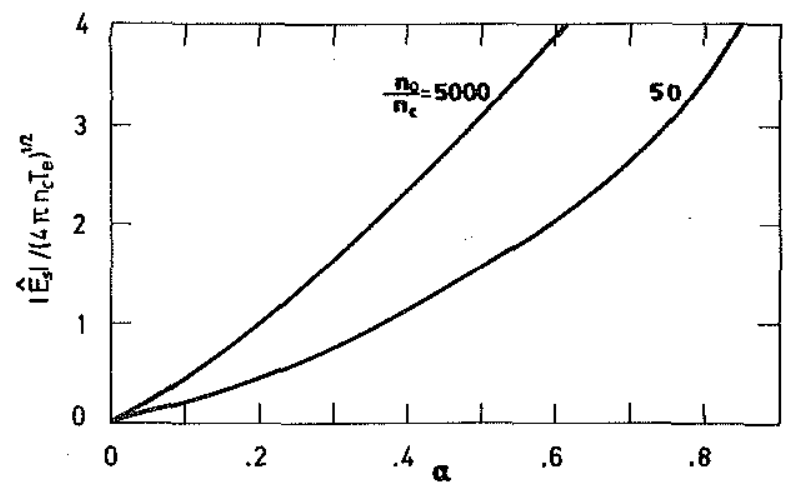

FIG. 3. Threshold electric field $\left|\hat{E}_{s}\right|$ (at the underdense, standing wave maximum) for a shock to exist, when $T_{e}$ $\sim t^{\alpha} ; n_{c}$ and $n_{0}$, critical and undísturbed densities.

ments ${ }^{9}$ and (planar) simulations, ${ }^{5}$ interpreted as plateaus, show nonnegligible density gradients (naturally weaker than the steep gradient in the critical layer). A shock should form in the underdense region to again make the flow subsonic, as required in the far expansion tail [Fig. 1(c)]; this shock, however, would be mixed up with the density oscillations in the underdense flow, which also make a WKB approximation inadequate (we have just finished an analysis of that region ${ }^{16}$ ). The entire (subsonic) overdense flow is affected by the steep transition (for $d T_{e} / d t<0$ the flow upstream of the shock, being supersonic, is unaffected). As the subsonic flow adjusts to $T_{B}$ variations, wave pockets may be cut out from the incident field, forming cavities; ${ }^{8,9}$ and we did find that cavities may form in subsonic flow. Clearly, when $T_{e}$ ceases to increase, a plateau may set up; such time developments have been observed in simulations. ${ }^{1,8,10}$

Important aspects of the physical problem which have been left out in our analysis are the existence of two electron temperatures and a substantial absorption within the critical layer (as, for instance, for $p$ polarization).

\section{ACKNOWLEDGMENT}

This work was supported by the Junta de Energia Nuclear of Spain.

${ }^{1}$ K. G. Estabrook, E. J. Valeo, and W. L. Kruer, Phys. Kluids 18, 1151 (1975).

${ }^{2}$ R. E. Kidder, in Proceeding of the Japan-U.S. Seminar on Laser Interaction with Matter, edited by C. Yamanaka (Tokyo International Book Company, Tokyo, 1973), p. 331; P. Mulser and C, van Kessel, Phys. Rev. Lett. 38, 902 (1977).

${ }^{3}$ C. E. Max and C. F. McKee, Phys. Rev. Lett. 39, 1336 (1977).

${ }^{4}$ K. Lee, D. W. Forslund, J. M. Kindel, and E. L. Lindman, Phys. Fluids 20, 51 (1977).

5J. Virmont, R. Pellat, and A. Mora, Phys. Fluids 21, 567 (1978).

${ }^{6}$ D. W. Forslund, J. M. Kindel, K. Lee, and E. L. Lindman, Phys. Rev. Lett. 36, 35 (1976).

${ }^{7}$ R. Fedosejevs, M. D. J. Burgess, G. D. Enright, and M. C. Richardson, presented at the 9th Annual Conference on Anomalous Absorption of Electromagnetic Waves, University of Rochester, New York (1979).

${ }^{8}$ H. C. Kim, R. L. Stenzel, and A. Y. Wong, Phys. Rev, Lett. 33, 886 (1974); T. P. Donaldson and I. J. Spalding, Phys. Rev. Lett. 36, 467 (1976).

${ }^{9}$ D. T. Attwood, D. W. Sweeney, J. M. Auerbach, and P. H. Y. Lee, Phys. Rev. Lett. 40, 184 (1978).

${ }^{10}$ D. W. Forslund, J. M. Kindel, K. Lee, E. L. Lindman, and R. L. Morse, Phys. Rev. 11A, 679 (1975); J. S. DeGroot and J, E. Tull, Phys. Fluids 18, 672 (1975).

${ }^{11}$ L. Spitzer, Jr., Diffuse Matter in Space (Interscience, New York, 1968), p. 184.

${ }^{12} \mathrm{~J}, \mathrm{R}$. Sanmartín and A. Barrero, Phys. Fluids 21, 1957 (1978); 21, 1967 (1978).

${ }^{13}$ L. D. Landau and E. M. Lifshitz, Fluid Mechanics (Pergamon, New York, 1959), Secs. 82 and 88.

${ }^{14}$ J. S. Pearlman and R. L. Morse, Phys. Rev. Lett. 40, 1652 (1978).

${ }^{15}$ J. A. Stamper, Phys, Fluids 18, 735 (1975); J. J. Thomson, C. E. Max, and K. G. Estabrook, Phys. Rev. Lett. 35, 663 (1975).

${ }^{16}$ J. R. Sanmartín and J. L. Montañés, J. Plasma Phys, 23, 349 (1980). 